\title{
Am Anfang war der Fuß
}

\section{Christian Heel}

\section{Vier Millionen Jahre Entwicklung stecken im Design des Fußes. Dementsprechend} ausgeklügelt ist sein System. Die Untersuchung und Therapie sollten aber nicht am Fuß stecken bleiben, da gerade das Zusammenspiel von Hüfte und Fuß für eine optimale Funktion entscheidend sein kann.

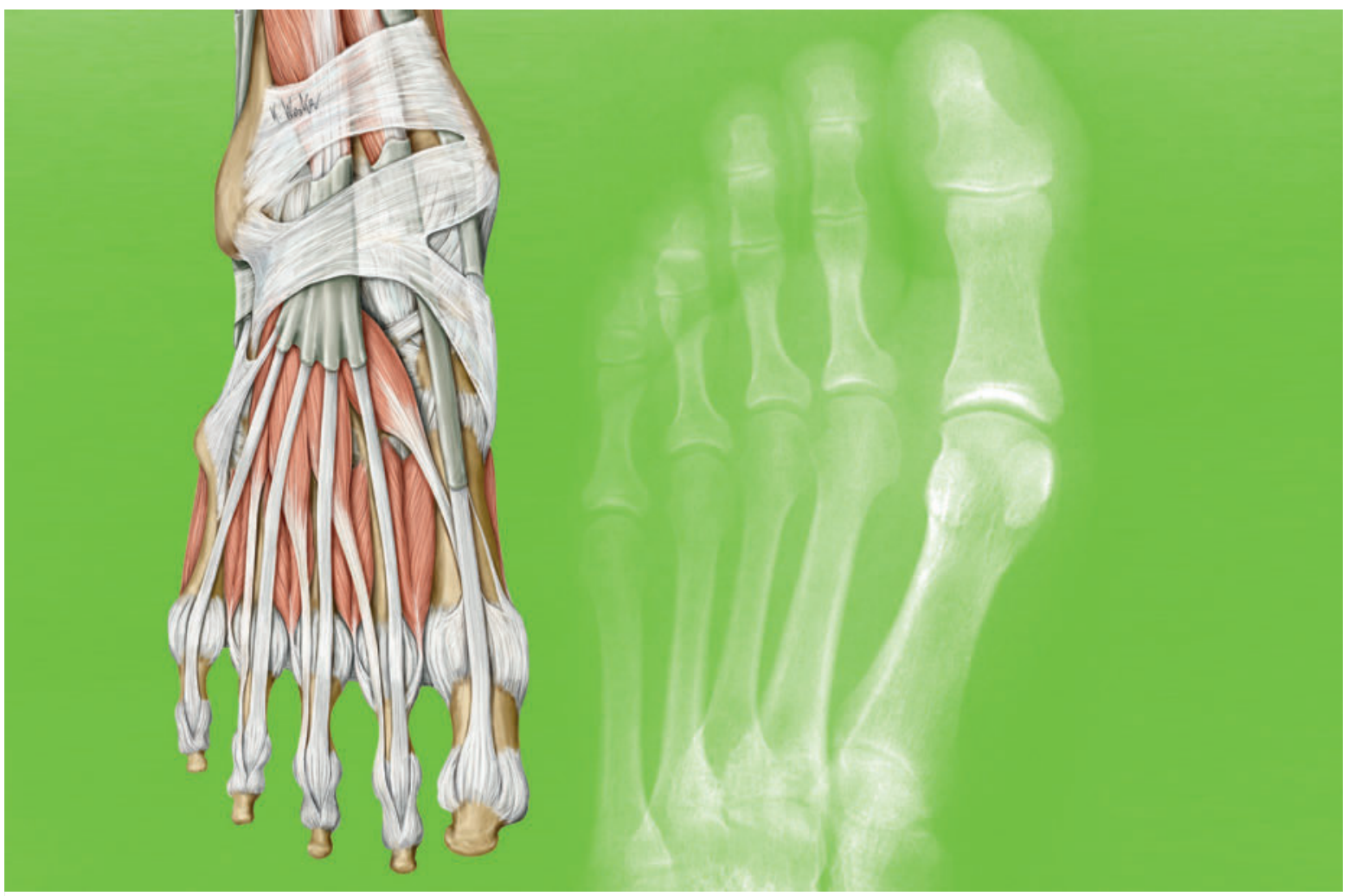

(Quelle: @ Prometheus. LernAtlas der Anatomie. Allgemeine Anatomie und Bewegungssystem. Grafik: Karl Wesker; aus: Schünke M, Schulte E, Schumacher U et al. Prometheus. LernAtlas der Anatomie. Allgemeine Anatomie und Bewegungssystem. Grafiken: M. Voll, K. Wesker. 5. Aufl. Stuttgart: Thieme; 2018)

Die Geburtsstunde des menschlichen Fußes: Vor mehr als vier Millionen Jahren begann die Aufrichtung zum Zweibeiner mit der Ausgestaltung des Fußes [1]. Die Entwicklung des Gehirns ist mit 1,5 Millionen Jahren dagegen vergleichsweise jung! 20 bis 25 Kilometer per pedes pro Tag entsprechen dem natürlichen Energieumsatz des Menschen. Dies führt zur Annahme, dass Menschen zu Zeiten der Wildbeuter täglich etwa sechs Stunden gegangen sind. 2017 berichtete der Spiegel über eine in dem Journal Nature veröffentlichte Studie der Universität Stanford, die das Gehverhalten von 700000 Probanden untersuchte: Der Durchschnitt geht heute 4900 Schritte pro Tag, das sind zirka drei Kilometer [2][3]. Selbst dieses eher gemüt- liche Gangpensum führt uns im Lauf des Lebens zwei Mal um die Erde.

Beim Joggen wird pro Laufkilometer jedes Bein mit zirka 60 Tonnen belastet, bei einem Marathon ergeben sich so etwa 2500 Tonnen pro Fuß [4]! Dies ist eine eindrückliche Leistung, die Präzision im Zusammenspiel von Statik und Dynamik, von muskulärer Spannung und Bewegungssteuerung erfordert. Die Grundlage dafür liegt in der optimierten Anlage der Anatomie. Die Bedeutung anatomisch korrekter Bewegung ist offensichtlich: 40 Prozent der Überlastungsschäden bei Läufern resultieren aus „anatomischen Auffälligkeiten“ [5], orthopädische 


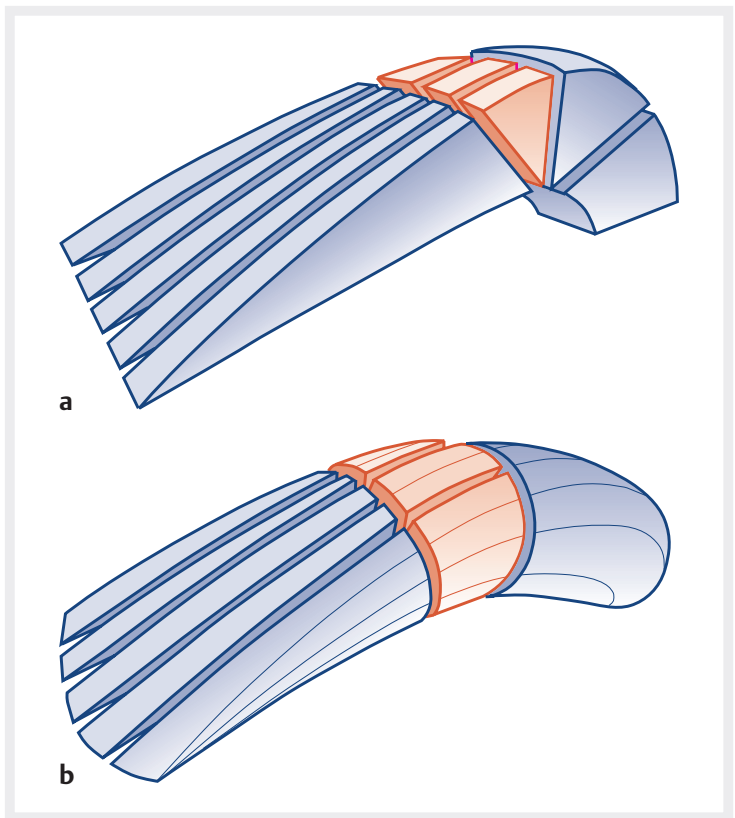

-Abb. 1 Keilprinzip und Spiralprinzip sorgen für eine lebenslange Belastungsstabilität. a Keilprinzip, b Spiralprinzip. (Quelle: Larsen C. Füße in guten Händen. 3. Aufl. Stuttgart: Thieme; 2014)

Fußbeschwerden und fußchirurgische Eingriffe sind entsprechend häufig.

\section{Das Fußskelett - ein architektonisches Meisterwerk}

Die Aufrichtung vom Vierbeiner zum Homo erectus erhöht die Anforderungen an die Füße. Der hohe labile Schwerpunkt lässt die Standbeinphase auf einem Bein zum Balanceakt werden, die kleine Standfläche der Sohle sucht Stabilität und in der Dynamik sind Stoßdämpfung und Abstoßkraft gefordert.

\section{Der Trick mit der Spirale}

Architektonisch erkennt man in der Anordnung der Fußknochen eindeutig die Form einer Spirale ( - Abb. 2). Die Ferse steht vertikal, der Vorfuß horizontal, Rückfuß und Vorfuß sind 90 Grad torquiert. Gleichzeitig zeigen Vor- und Rückfuß einen nach oben konvexen Oppositionsbogen (C-Bogen). Von oben betrachtet ergibt sich das Bild eines flachen S-Bogens. Torsion, C-Bogen und S-Bogen sind die Merkmale der 3D-Geometrie des Fußes ( $\triangleright$ Abb.3). Das „Herzstück“ der Fußknochen sind die Ossa cuneiformia, die keilförmig im Zentrum des Fußskeletts sitzen. Durch die spiralige Verschraubung verkeilen Cuneiformia II + III - das Längsgewölbe erreicht dadurch eine beachtliche knöcherne Stabilität. Die Bedeutung der Cuneiformia ähneln der des Schlusssteines beim Bau einer Gewölbekuppel.

Beim Fersenlot macht das Zusammenspiel von Hüftgelenk und Vorfuß den Unterschied für die Stabilität des Fußge-

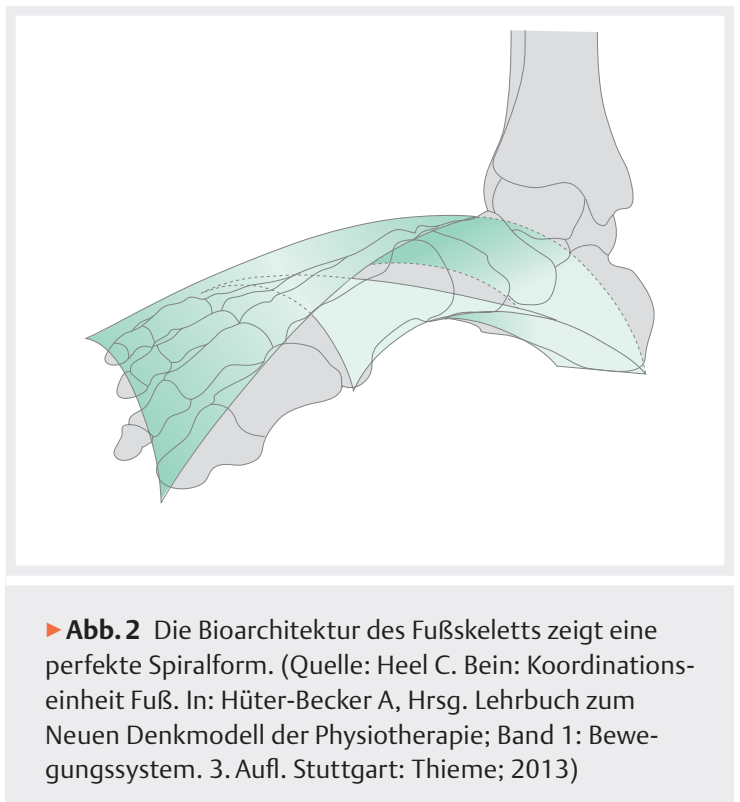

wölbes. Dreht der Vorfuß nach innen im Sinne einer Pronationsbewegung und das Femur im Hüftgelenk nach außen, richtet sich das Bein axial aus, das Knie zeigt orthograd nach vorne. Auch hier tritt dasselbe Konstruktionsprinzip auf. Die Drehrichtungen sind grundlegend und in der Anordnung vieler anatomischer Strukturen sichtbar. Die Form des Femurs, die Anlage der Kreuzbänder oder der Verlauf der Mm. iliopsoas, sartorius und tibialis anterior zeigen die Form einer Helix ( $>$ Abb. 4). Die axiale Ausrichtung des Beines sorgt für eine zentrierte Belastung auf den Talus, das Fersenbein steht im Lot - Markenzeichen einer stabilen Fuß- und Beinsituation.

\section{Rotationstests}

Die Torsion von Bein- und Fußknochen entsteht primär durch eine gegensinnige Rotation. Die Konsequenz: Hüftgelenk, Kniegelenk und Fußwurzelgelenke brauchen ein gewisses Rotationsspiel, das jeweils getestet werden sollte.

\section{Ausführung der Rotationstests}

1. Hüftrotation: in Bauchlage, Idealwerte in Extension des Hüftgelenks: Außenrotation 50, Innenrotation $40^{\circ}$, Grenzwert, um kompensationsfrei gehen zu können: je $30^{\circ}$ [4].

2. Pronation des Vorfußes: Das größte Bewegungsausmaß findet im Talonavikulargelenk und im Gelenk zwischen Os naviculare und Os cuneiforme mediale statt. Das Bewegungsausmaß zwischen Os calcaneus und Os cuboideum ist deutlich geringer. Getestet wird mit proximaler Fixierung die Bewegung der jeweils distalen Gelenkpartner. In Summe sollte $15-20^{\circ}$ reine Pronation im Vorfuß bei fixierter Ferse möglich sein [4]. Im Unterschied dazu ist das Gesamtausmaß der Pronations- und Supinationsbewegung ohne fixierte Ferse deutlich größer $\left(35^{\circ} / 0^{\circ} / 50^{\circ}\right)[6]$. 


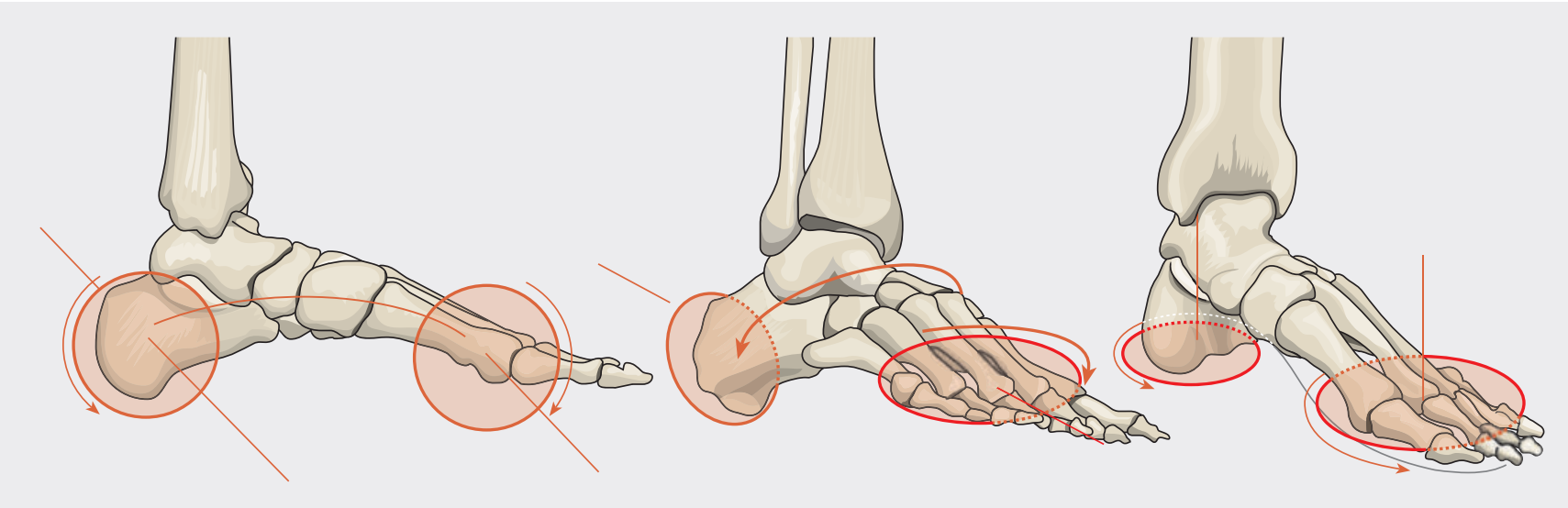

Abb. 3 3D-Geometrie des Fußskeletts: C-Bogen, Torsion und S-Bogen sind Merkmale der spiraligen Verschraubung des Fußes. Daraus resultiert ein Zug von dorsolateral an der Ferse über den Fußrücken nach ventromedial zum Großzehengrundgelenk. Fersenaußenseite und Großzehenballen sind die Zonen, die in der Standbeinphase am stärksten belastet werden. Die der Spirale innewohnende Dehnspannung sorgt für Stabilität und gleichmäßige Belastungsverteilung. (Quelle: C. Heel; graf. Umsetzung: Thieme Gruppe)

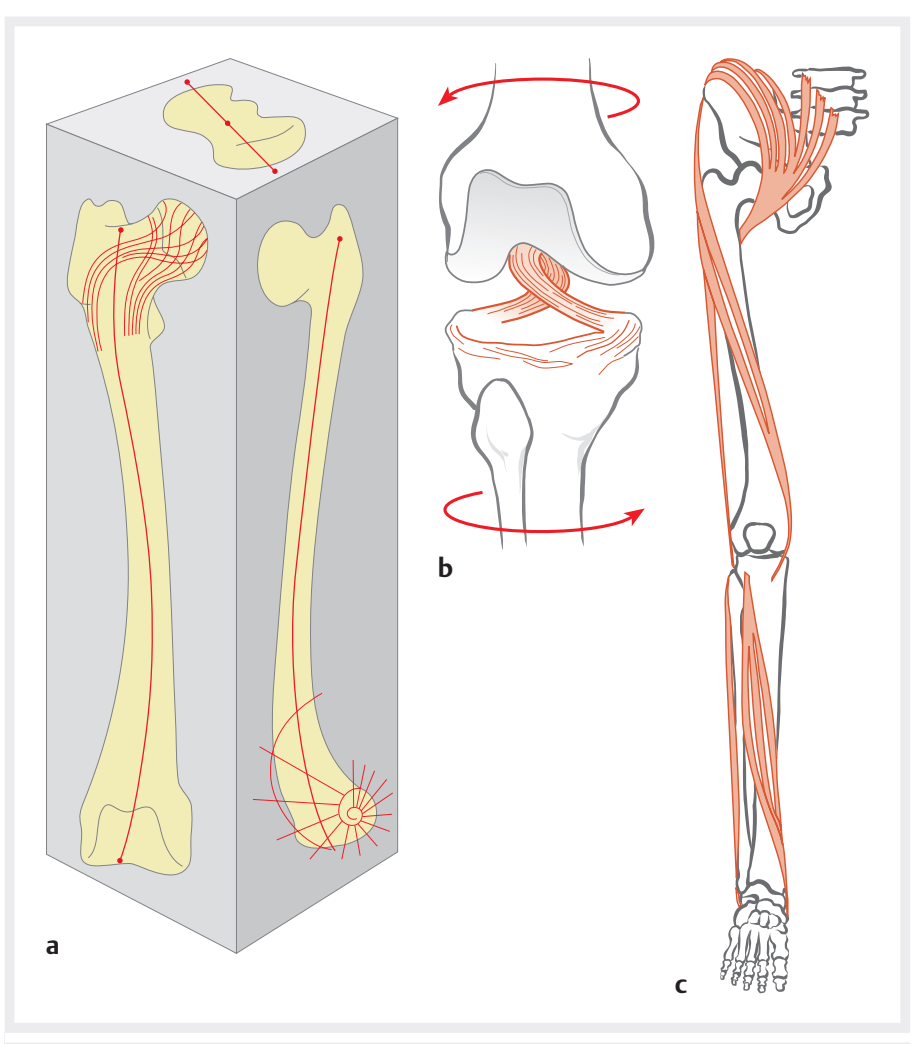

Abb. 4 Spiralform anatomischer Strukturen. a Femur: Röhrenknochen mit Spiralform - Torsion, C-Bogen und S-Form sind Merkmale für eine Schraubenspirale. (Quelle: Heel C. Bein: Koordinationseinheit Hüftgelenke. In: Hüter-Becker A, Hrsg. Lehrbuch zum Neuen Denkmodell der Physiotherapie; Band 1: Bewegungssystem. 3. Aufl. Stuttgart: Thieme; 2013) b Die Anlage der Kreuzbänder zeigt die Form einer Helix. c Der Verlauf der Mm. iliopsoas, sartorius und tibialis anterior zeigt die Form einer Helix. (Quelle: Larsen C. Füße in guten Händen. 3. Aufl. Stuttgart: Thieme; 2014)

3. Innenrotation der Tibia im Kniegelenk: Das Kniegelenk lässt nur in Beugestellung eine Rotation zu. Die Innenrotation lässt sich am besten im Sitzen mit hängendem Unterschenkel testen. Dabei wird der Unter- schenkel nach innen gedreht, als Referenzpunkt für die Einschätzung der Beweglichkeit dient die Tuberositas tibiae. Mindestens $10^{\circ}$ Innenrotation sollte möglich sein.

\section{Einbeinstand - einfacher Funktionstest mit hoher Aussagekraft}

Der Einbeinstand erfordert eine gute Organisation der Bein- und Fußstatik. Zusätzlich lassen sich in dieser Position die Lastübertragung vom Rumpf aufs Bein anhand der Beckenstellung sowie die sensomotorischen Fähigkeiten, das Gleichgewicht zu organisieren, beurteilen. Abweichungen vom Ideal werden im Vergleich zum Zweibeinstand meist deutlicher und damit besser sichtbar. Bewegungsdefizite und eine mangelhafte Aktivierung und Koordination der posturalen Muskulatur werden offensichtlich.

Bei einer eingeschränkten Pronationsbeweglichkeit des Vorfußes kippt der Rückfuß nach medial, im Sinne eines Pes valgus, oft kombiniert mit einer nach innen gedrehten Oberschenkelstellung (Kneeing in, Beinvalgus). Umgekehrt kann eine mangelhafte Außenrotation im Hüftgelenk dasselbe Phänomen bewirken ( $\triangleright$ Abb. 6). Beides ist oftmals kombiniert mit einer schlechten Balance. Legt man in diesem Fall ein Holzplättchen unter die Großzehe und den Großzehballen des Patienten, um der verminderten Vorfußpronation entgegenzuwirken, verbessert sich die Gleichgewichtssituation meist spontan. Ein Testen der Pronationsbeweglichkeit, wie oben beschrieben, bestätigt dann das erwartete Pronationsdefizit.

X-Beinhaltung und Knickfuß finden sich allerdings auch ohne Einschränkung der beschriebenen Rotationskomponenten, was auf eine insuffiziente Ansteuerung der posturalen Muskulatur hinweist. Die Leitstrukturen hierfür sind die tiefen Außenrotatoren im Hüftgelenk: Mm. quadra- 


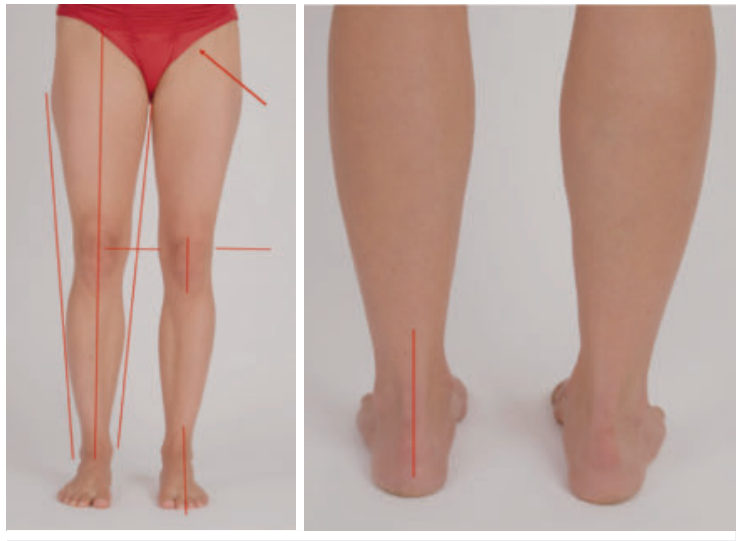

Abb. 5 Blickdiagnose - Kriterien im Stand. (Quelle: Spiraldynamik AG)
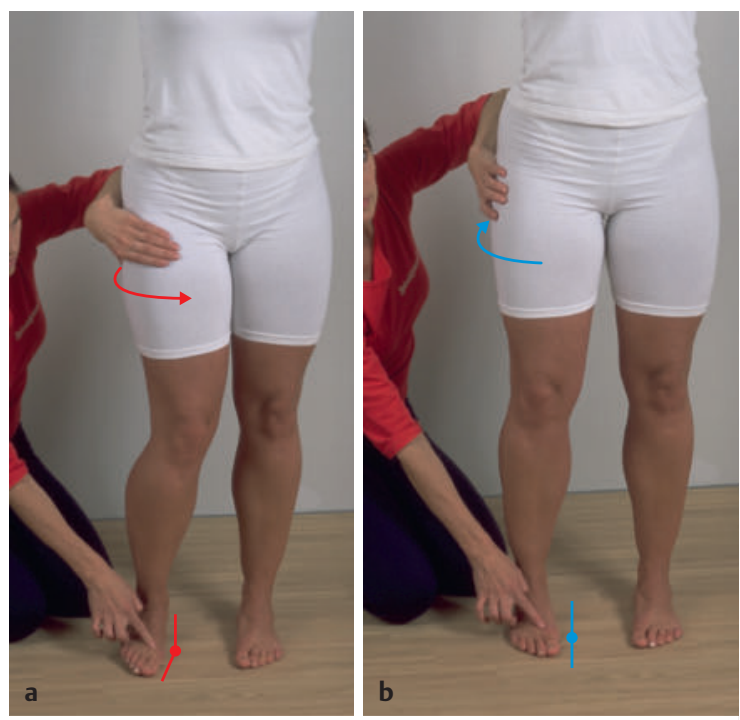

Abb. 6 Das Spiralprinzip mit den definierten Drehrichtungen, Oberschenkel nach außen, Unterschenkel nach innen, ist grundlegend für eine axial verlaufende Beinachse. Der funktionelle Zusammenhang zwischen Femur-Außenrotation und Vorfuß-Pronation sollte in der Therapie aktiv geübt werden. a Ausgangshaltung, b korrigierte Haltung. (Quelle: Larsen C. Füße in guten Händen. 3. Aufl. Stuttgart: Thieme; 2014)

tus femoris, obturatorii, gemelli und piriformis. Sie sorgen für die rotatorische Stabilität des Femurs. Der M. peroneus longus liefert die aktive Gegenbewegung im Vorfuß. Das Zusammenspiel dieser beiden Muskelsysteme ergibt eine stabile Bein- und Fußachse, die auch durch die Übertragung der Rumpflast auf das Bein beeinflusst wird. Die Stellung des Beckens entscheidet, ob die Rumpflast zentriert auf das Bein übertragen wird oder nicht. Der Körperschwerpunkt verlagert sich auf die Standbeinseite, indem das Becken über die kleinen Glutaeen aktiv seitlich nach kaudal gezogen wird, entsprechend einer proximalen Abduktion im Hüftgelenk ( $\triangleright$ Abb. 7).
BLICKDIAGNOSTIK - KRITERIEN IM STAND ( $\triangle A B B .5$ )

- Beckenhaltung aufrecht, Hüftgelenk gestreckt

- Bein axial, Innenseite und Außenseite etwa gleich lang

- Oberschenkel Rotation minimal nach außen gehalten

- Femurkondylenquerachse liegt in der Frontalebene

- Tuberositas tibiae zentriert

- Patella orthograd

- Ferse lotrecht

- Großzehenballen am Boden verankert

- Fuß gerade nach vorne (abhängig von den Knochentorsionen)

\section{Balance: Spannung im Gleichgewicht}

Die propriozeptiven Fähigkeiten für Balance, Adaptionsfähigkeit und Reaktionsfähigkeit hängen primär von zwei Faktoren ab: der optimierten statischen Ausrichtung und einer homogenen Spannungsverteilung. Dabei bedingt das eine das andere. Je besser die Statik, desto geringer die geforderte Haltespannung, was die Agilität und Reaktionsfreudigkeit sämtlicher Muskeln erhöht. Die Spannungsverteilung im Faszien- und Bandgewebe ist entscheidend. Am Fuß verläuft die Hauptzuglinie dorsal vom Großzehengrundgelenk über den Fußrücken zur Fersenaußenseite. Faszien und Bänder sind entlang dieser Linie gut aufgespannt, dies gilt vor allem auch für die Retinakuli und die lateralen Sprunggelenkbänder ( $>$ Abb. 8). Sind die passiven Strukturen homogen gespannt, werden Spannungsunterschiede von den Golgiapparaten schneller erfasst und reflektorisch verarbeitet. Mehr Input führt zu differenzierterem Output. Die Gleichgewichtsfähigkeit verbessert sich signifikant. Auf einem Bein stehen wird zum Kinderspiel.

\section{ÜBUNGSTIPP I}

Beim Zähneputzen 2x je 1 Minute auf einem Bein stehen mit axialer Ausrichtung im Bein und aufgerichtetem Fußgewölbe [7].

\section{Knickfuß und Supinationstrauma - eine häufige Kombination?}

Beim Pes valgus sind die lateralen Seitenbänder zu locker. Gewohnheitsmäßig knickt das Bein nach innen, ohne spezifische Stützaktivität der peronealen Muskulatur. Die Großzehenballe wird passiv auf den Boden gepresst. Im Falle eines Fehltritts wird zuerst das lockere Seitenband beschleunigt. Wertvolle Millisekunden verstreichen, bis die Spannung des lateralen Bandtraktes zunimmt und die Golgiapparate ihre Signale Richtung Rückenmark schicken. Ist nun die efferente Antwort, das blitzschnelle Aktivieren der peronealen Muskulatur wenig geübt, gibt es auch hier eine Verzögerung. Die Krafteinwirkung auf die Bänder ist mittlerweile zu groß und die Ruptur die unausweichliche Folge. 

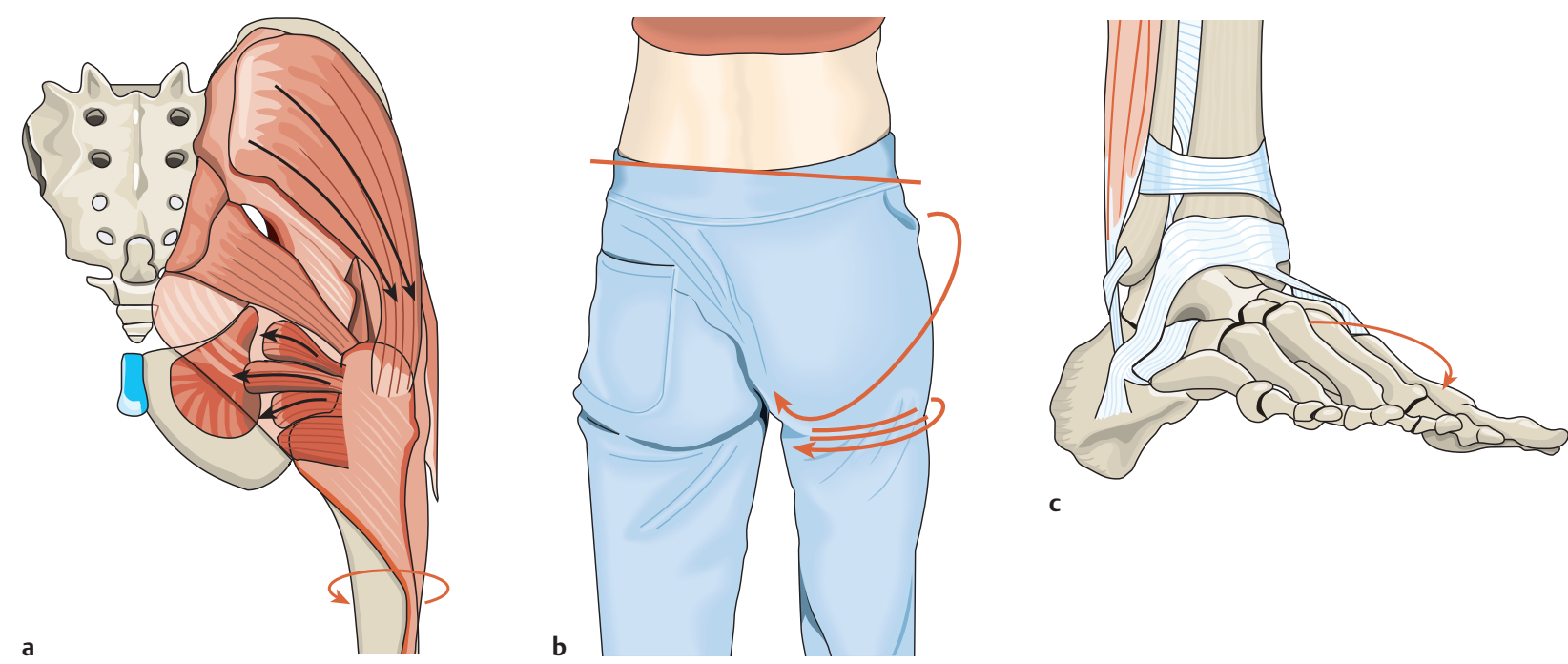

-Abb.7 Zusammenspiel der Muskulatur beim Einbeinstand. a Die tiefen Außenrotatoren verlaufen fächerförmig unter der Gesäßmuskulatur. Sie wirken rotationsstabilisierend auf das Femur. Die kleinen Glutaeen ziehen das Becken standbeinseitig tief und bringen den Körperschwerpunkt näher ans Bein. b In der Landephase (Loading Response) werden die tiefen Außenrotatoren impulsartig aktiviert. Das Becken bewegt sich spiralförmig über das Bein. c Der M. peroneus longus dreht den Vorfuß nach innen (Pronation) und wirkt damit der Außenrotationskraft der Hüfte entgegen. (Quelle: C. Heel; graf. Umsetzung: Thieme Gruppe)

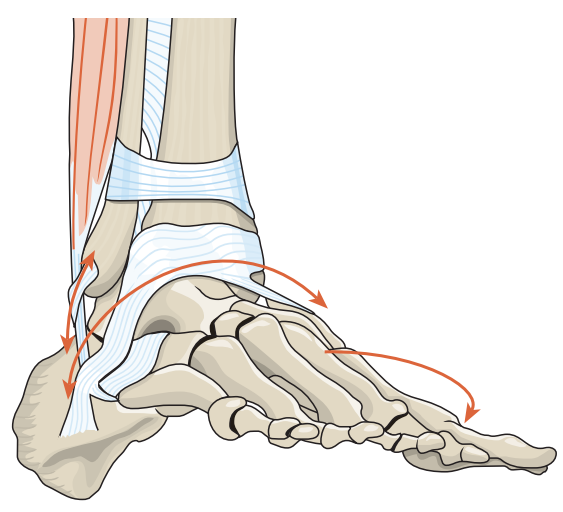

Abb. 8 Die spiralige Verschraubung von Vor- und Rückfuß führt zur Dehnspannung des Retinakulums und der Außenbänder am oberen Sprunggelenk. Sämtliche Strukturen auf der Linie von der Fersenaußenseite zum Großzehengrundgelenk kommen unter Zug. (Quelle: C. Heel; graf. Umsetzung: Thieme Gruppe)

\section{ÜBUNGSTIPP II}

Den Vorfuß resistiv gegen Widerstand pronieren und damit Ansteuerung und Kraft des M. peroneus longus üben [8] (• Abb.9).

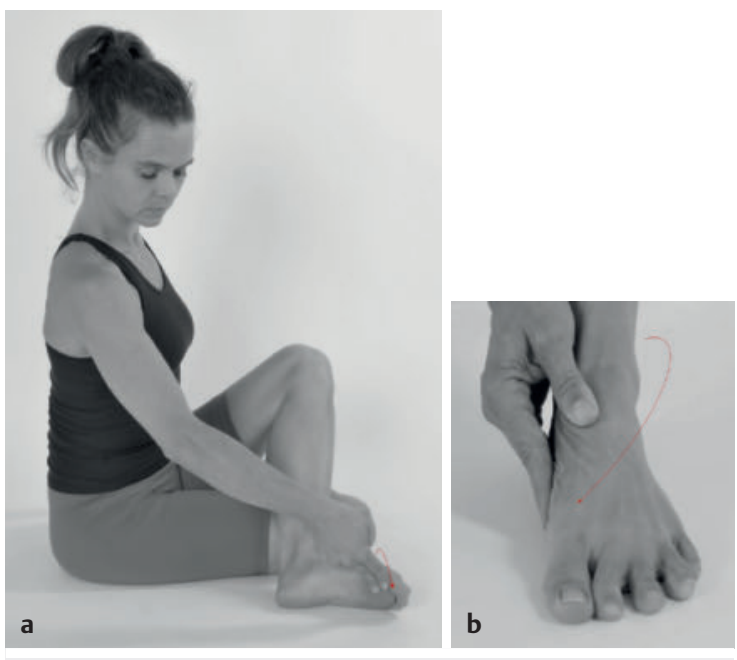

Abb. 9 Das differenzierte Aktivieren der Peronealmuskulatur kombiniert mit der Vorfußpronation lässt sich resistiv gegen den Widerstand der Finger oder des Therabandes gut üben. Setzen Sie sich bequem auf den Boden oder Stuhl und achten Sie auf die isolierte Vorfußpronation. Ferse und Knie bleibt nach außen gedreht und bewegen nicht. a Widerstand am Großzehenballen gegen die Pronation im Schneidersitz. b Auch mit aufgestelltem Fuß lässt sich die Aktivierung der Peronealmuskulatur üben. (Quelle: Spiraldynamik AG) 


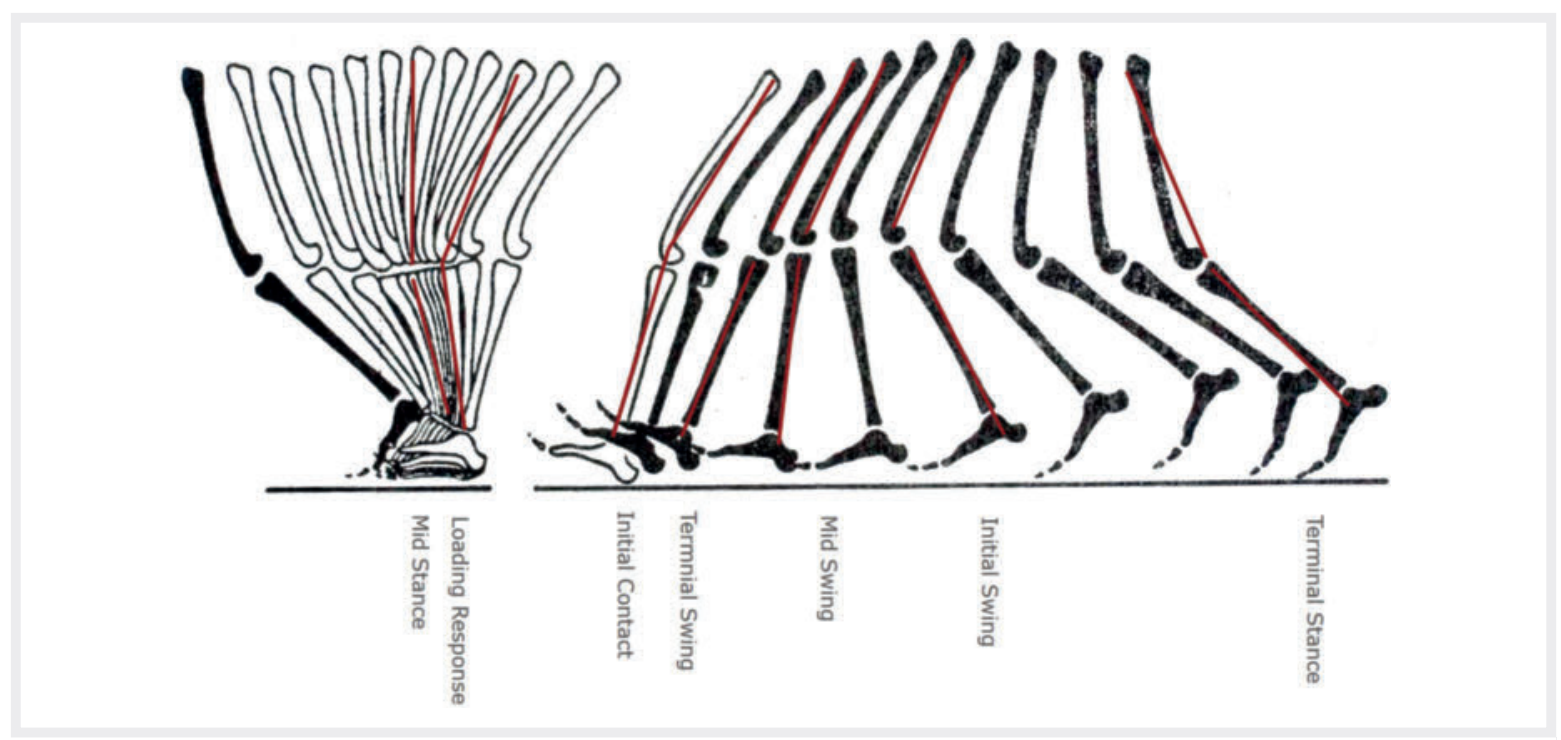

Abb.10 Die Gangzyklen nach dem Rancho Los Amigos System. (Quelle: Spiraldynamik AG)

\section{Stoßdämpffunktion - das Einmaleins des Raubkatzengangs}

Die Beschwerden oder Erkrankungen, die durch eine verbesserte Stoßdämpfwirkung beim Gehen positiv beeinflusst werden, sind vielfältig: Gelenkarthrose der Fuß- und Beingelenke, Facettenarthrose der Lendenwirbelsäule, Meniskopathien, Diskopathien, Iliosakralgelenkschmerzen und sämtliche Symptome, die durch Rigidität und Elastizitätsverlust in den Weichteilen resultieren, wie die Plantarfasziitis beim Fersensporn oder die Achillodynie. Selbst bei der Stressinkontinenz kann die Stoßdämpfung ein wichtiges Puzzleteil sein.

\section{Die Gangphasen}

Die Standbeinphase lässt sich vom zeitlichen Ablauf genau unterteilen ( $\mathbf{A}$ bb. 10). Beim Gehen setzt der Fuß in der Regel mit der Ferse, dem rund geformten Tuber calcanei auf. Der harte Fersenaufprall ist ein valides Zeichen einer mangelnden Stoßdämpfung. Ein gestrecktes bis überstrecktes Kniegelenk und ein stark ausgeprägter Pelvic Drop (proximale Adduktion der Standbeinhüfte) sind meist ursächlich dafür. Nach dem Initial Contact [9] folgt die zweite Phase im Standbeinzyklus: Loading Response der Moment der Stoßdämpfung.

\section{Ausgeklügelte Mechanik und einfache Bewegungssteuerung}

In der Landephase rollt der Fuß über den runden Tuber calcanei nach vorne, bis der Vorfuß und damit die gesamte Sohle Bodenkontakt hat. Der Unterschenkel beugt ein wenig nach vorne, das Knie steht leicht vor dem Sprunggelenk. Im Knie entsteht eine leichte Flexion, im oberen Sprunggelenk eine leichte Dorsalextension. Sind Querund Längsgewölbe genügend aufgespannt, können diese federelastisch nachgeben ( $\mathbf{A}$ Abb. 11). In diesem zeitlich sehr kurzen Moment greifen unterschiedliche Mechanismen für eine perfekte Stoßdämpfung ineinander:

1. Der knöcherne Gewölbebogen des Vorfußes

( Abb. 11a) befindet sich im distalen Fußwurzelbereich, die drei Ossa cuneiformia bilden zusammen mit dem Os cuboideum einen perfekten Bogen, die Ossa cuneiformia II \& III verkeilen sich im Gewölbebogen und sorgen für knöcherne Stabilität. Das Kuboid und das Os cuneiforme mediale organisieren sich in einem Oppositionsbogen.

2. Die spiralige Verschraubung des Fußes unterstützt den Quergewölbeaufbau. Der supinatorisch orientierte Kalkaneus dreht das Kuboid mit in diese Richtung, die pronatorische Vorfußbewegung ist identisch mit der Einrollbewegung von Metatarsale I und Os cuneiforme mediale. Die Leitmuskulatur der Fußspirale, M. tibialis posterior und M. peroneus longus, organisieren auch das Quergewölbe ( $\mathbf{A b b} \mathbf{1 1 \mathbf { 1 }}$ )

3. Auf die richtige Spannung und Kraft kommt es an, der Rest ist reine Mechanik. Die beiden genannten Muskeln wirken im Sprunggelenk plantarflektorisch. Im Moment des Initial Contacts erhöht sich die Muskelspannung, ähnlich einer isometrischen Aktivität, die Bewegung der Tibia nach vorne verlängert die Mm. tibialis posterior und peroneus longus während der Phase Loading Response und überträgt damit den Zug mechanisch auf die Insertionsstellen. Es ist, als ob die Muskeln wie straffe Seile an den Knochen ziehen und das Gewölbe formen. Allerdings ist es nicht wie bei Stahlseilen, denn die Kollagenanteile der Muskelsehnen und der Titinfilamente geben geringfügig elastisch nach, was das Fußgewölbe federn lässt. Die Federung funktioniert mechanisch und nicht neurologisch. Die Muskeln geben nicht exzentrisch nach. 


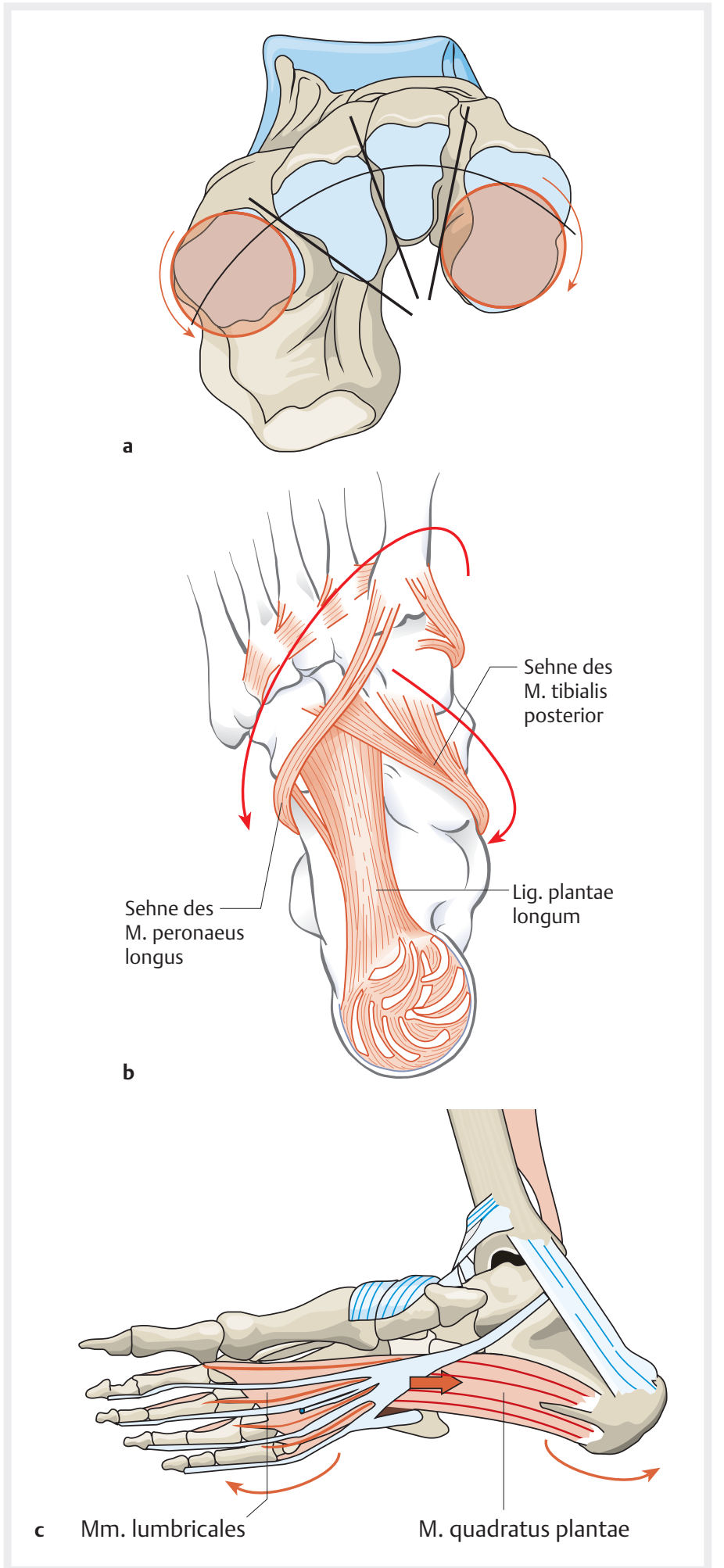

- Abb.11 Mechanismen für eine perfekte Stoßdämpfung. a Gewölbebau in der distalen Fußwurzelreihe. (Quelle: C. Heel; graf. Umsetzung: Thieme Gruppe) b Mm. peroneus longus und tibialis anterior unterstützen den Quergewölbeaufbau. (Quelle: Larsen C. Füße in guten Händen. 3. Aufl. Stuttgart: Thieme; 2014) c Mm. quadratus plantae und lumbricales sind über die Sehne des M. flexor digitorum longum verbunden. Die Kraft des M. quadratus plantae lässt sich effektiv auf die das Quergewölbe stabilisierenden Mm. lumbricales übertragen. (Quelle: C. Heel: graf. Umsetzung: Thieme Gruppe)
4. Derselbe Mechanismus findet mehrfach statt. Der M. quadratus plantae sitzt unter der Ferse an der Fußsohle und inseriert in die Sehne der langen Zehenbeuger ( $\triangleright$ Abb. 11c). Vis-à-vis der Insertionsstelle entspringen aus den Sehenbifurkationen die Mm. lumbricales. Diese verstreben das Quergewölbe muskulär. Entwickelt die tiefe Sohlenmuskulatur beim Landen eine gewisse Steifigkeit, überträgt sich die Längsspannung der Fußsohle auch auf das Quergewölbe. Auch hier gibt es wieder elastische Kollagenstrukturen, die federnd nachgeben können.

5. Die Dorsalextension im oberen Sprunggelenk des Standbeins verlängert beim Landen die Achillessehne und gibt Zug auf den Triceps surae. Ist dieser auch wieder isometrisch gespannt, wirkt die Zugkraft des Triceps surae auf das Fersenbein und unterstützt dort die supinatorische Aufrichtung. Ein Geheimtipp bei vielen Knickfüßen ist die Stoßdämpferbewegung: Knie ein wenig vor das Sprunggelenk beugen lassen, die Wadenmuskulatur adäquat spannen. Dies wirkt oft erstaunlich positiv auf die Aufrichtung eines Pes valgus.

\section{ÜBUNGSTIPP III FÜR PATIENTEN}

Rollen Sie mit ihren Händen das Os cuneiforme mediale und das Kuboid jeweils zusammen mit den Metatarsalknochen I und V zu einem Oppositionsbogen und mobilisieren Sie so Ihr Quergewölbe [10].

\section{ÜBUNGSTIPP IV FÜR PATIENTEN}

Trainieren Sie ihre Mm. lumbricales ( Abb. 12). Stellen Sie im Sitzen den Vorfuß auf die Ballen, die Ferse etwa vier Zentimeter vom Boden angehoben ( $\triangleright$ Abb. 12a). Denken Sie nun an die Einrollbewegung des Quergewölbes und beginnen Sie, die Zehenkuppen in den Boden zu drücken, die Grundgelenke werden etwas angehoben, die Zehen bleiben lang, die Ferse sinkt zwei Zentimeter nach unten ( $\triangleright$ Abb. 12b). Die Bewegung erinnert an den „Raupengang“. Der Vorfuß zieht den Rückfuß etwas nach vorne. So entwickeln Sie Vorfußkraft und üben die Abstoßbewegung [4].

\section{Die drei wichtigsten Parameter eines elastischen Gan-} ges:

- anatomisch sinnvolle Ausrichtung der Fußgewölbe und Beinachse integriert in die korrekten Abfolgen der Standbeinphase

- Elastizität und Kraft in Bindegewebe und Muskeln

- Fähigkeit, die Muskelspannung schnell und adäquat anzupassen 


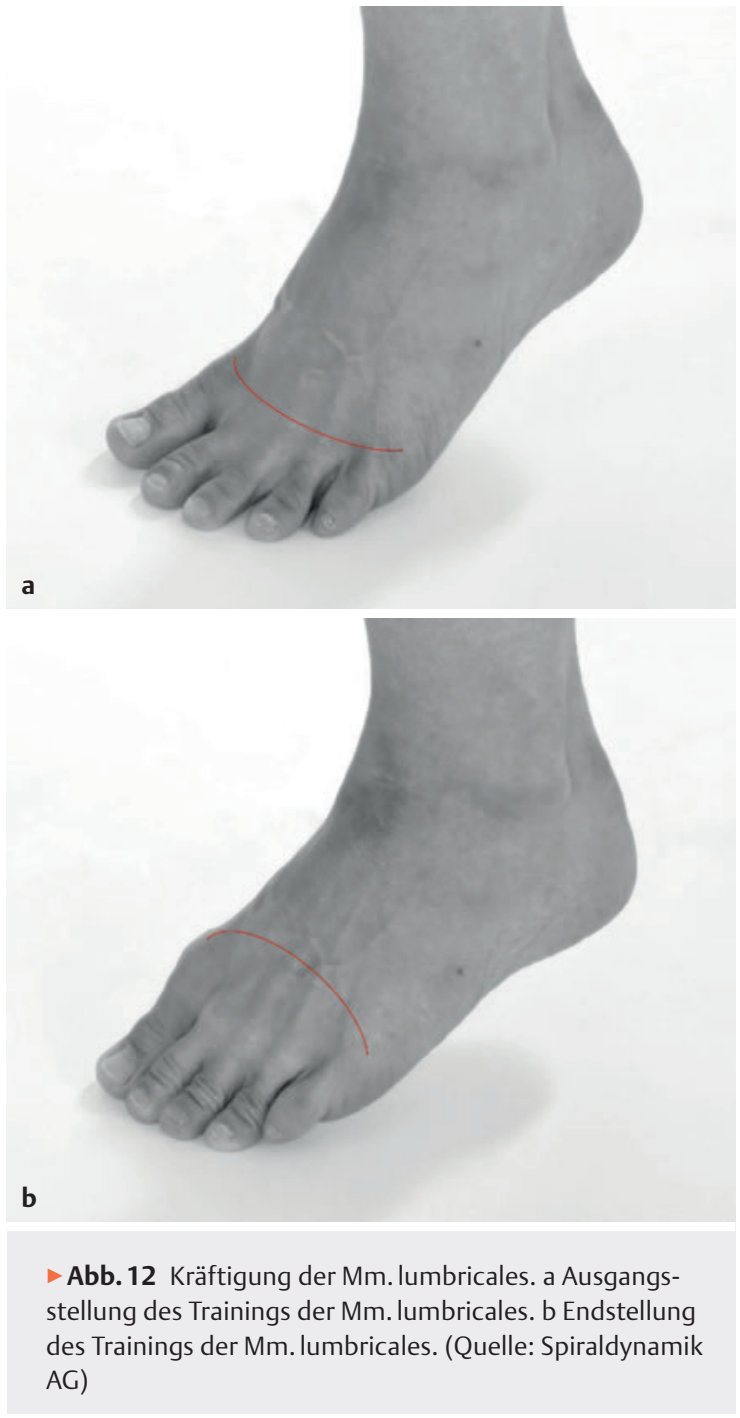

\section{Fazit: Functional Reasoning}

Die funktionellen Zusammenhänge sehen und verstehen zu können, ermöglicht es dem Therapeuten, die Ursachen vieler Beschwerdebilder zu entdecken. Sind die Ursachen erkannt, gilt es, die anatomischen Möglichkeiten abzuklären, um entweder das Problem zu eliminieren oder die günstigste Kompensationsstrategie zu erarbeiten.

\section{Die Therapieschritte auf den Punkt gebracht:}

1. Patienten verstehen ihre ungünstigen Gewohnheiten.

2. Patienten lernen achtsam, im Alltag Fehlbewegungen wahrzunehmen.

3. Patienten erarbeiten mittels aktiven Übungen die notwendigen körperlichen Voraussetzungen, wie selektive Mobilität oder differenzierten Krafteinsatz bestimmter Muskeln.
4. Patienten üben die anatomisch sinnvollen Bewegungsabläufe.

5. Patienten integrieren das Gelernte in ihren Bewegungsalltag.

Gehen gilt als die archaische Bewegung des Menschen. Bereits vor 2400 Jahren postulierte Hippokrates: „Gehen ist die beste Medizin“. Die Qualität des Gehens lässt sich ein Leben lang verbessern. Mit dem Üben des Gehens lassen sich sozusagen neue Aspekte der „Menschwerdung“ entdecken.

\section{Autorinnen/Autoren}

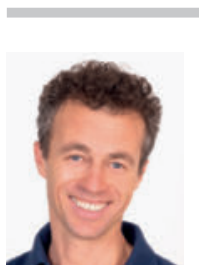

\section{Christian Heel}

Physiotherapeut, Spiraldynamik ${ }^{\circledR}$ Dozent und Leiter der Spiraldynamik Akademie. In den Bergen groß geworden, hat er sich schon früh intensiv mit sämtlichen Formen des Gehens und Laufens auseinandergesetzt. Als Therapeut und Spiraldynamik Dozent ist ihm dieses praxiserprobte Wissen heute zur Grundlage seines therapeutischen Handelns geworden. Er hat sich zum Ziel gesetzt, dies an Kolleginnen und Kollegen weiterzugeben, was er seit gut 25 Jahren mit viel Freude macht.

\section{Korrespondenzadresse}

Christian Heel
Spiraldynamik Akademie
Südstr. 113
8008 Zürich
Schweiz
christian.heel@spiraldynamik.com

Literatur

Literaturverzeichnis am Ende der HTML-Version unter www.thieme-connect.de/products/manuelletherapie

Bibliografie

DOI https://doi.org/10.1055/a-1213-0230

manuelletherapie 2020; 24: 160-167

(c) Georg Thieme Verlag KG Stuttgart · New York ISSN 1433-2671 